\title{
Detrimental consequences of the paracetamol tablet elastic relaxation during ejection
}

\begin{abstract}
Background: It is generally accepted that the tablet elastic relaxation during compaction plays a vital role in undermining the final tablet mechanical integrity. One of the least investigated stages of the compaction process is the ejection stage. Method: This work has successfully monitored the paracetamol tablet dimensional changes during ejection using noncontact dimensional measuring devices. The extent of the tablet damage was physically viewed by examining the presence of cracks on the tablet side surfaces upon complete ejection from the die cavity. Results: Damaged tablets were obtained when the paracetamol tablets exhibited comparatively high elastic relaxation during the ejection stage of the compaction process. Conclusion: Hence, this work presents evidences of the detrimental consequences of the paracetamol tablet elastic relaxation during ejection on its final mechanical integrity.
\end{abstract}

Keyword: Capping; Compaction; Ejection; Elastic relaxation; Lamination; Stored elastic energy 